\title{
Polyphenol-Rich Muscadine Grape Extract Reduces Triple Negative Breast Cancer Metastasis in Mice with Changes in the Gut Microbiome
}

\author{
Marianne Collard \\ Boston University
}

Nataleigh N Austin

Wake Forest University School of Medicine

Heather Brown-Harding

Wake Forest University

\section{Brian Westwood}

Wake Forest University School of Medicine

\section{E Ann Tallant}

Wake Forest University School of Medicine

Patricia E Gallagher ( $D$ PGALLAGH@WAKEHEALTH.EDU)

Wake Forest University School of Medicine https://orcid.org/0000-0003-1834-4189

\section{Research article}

Keywords: triple negative breast cancer, nutraceutical, gut microbiota, migration, proliferation, butyrate, metastasis

Posted Date: April 8th, 2021

DOI: https://doi.org/10.21203/rs.3.rs-384168/v1

License: (9) This work is licensed under a Creative Commons Attribution 4.0 International License. Read Full License 


\section{Abstract}

Background Triple negative breast cancer (TNBC) has a high propensity to metastasize and no treatments are available to slow or prevent metastatic progression. The goal of this study is to determine whether a proprietary high-polyphenol content muscadine grape extract (MGE) inhibits TNBC metastasis. Methods 4T1 TNBC cells were injected into the mammary fat pad of 6-week-old female Balb/c mice. After 2 weeks, tumors were surgically removed and mice were placed into a control $(n=8)$ or treatment group that received $0.1 \mathrm{mg} / \mathrm{mL}$ total phenolics MGE in the drinking water $(\mathrm{n}=8)$ for 4 weeks. Immunohistochemistry (Ki67, a-SMA) and hemotoxylin and eosin staining were used to quantify metastases. Gut microbial composition was determined by $16 \mathrm{~S}$ rRNA sequencing and short chain fatty acids (SCFAs) were detected by gas chromatography. MDA-MB-231, BT-549 and 4T1 TNBC cell motility and cytoskeletal organization was assessed in vitr o by scratch wound migration and confocal microscopy, respectively. Data were evaluated by student's t -test. Results MGE reduced metastatic proliferation in mouse lungs $(33.3 \%)$ and livers $(58.3 \%)$ and decreased the number $(51.1 \%)$ and size $(17.4 \%)$ of liver metastases, resulting in a $55.7 \%$ reduction in metastatic tumor burden $(P<0.01)$. Serum IL-6 was reduced $99.6 \%$ in MGE-treated mice $(P=0.06)$. MGE attenuated migration, altered cytoskeletal organization, and reduced RHAMM expression in TNBC cells $(P<0.05)$. The gut microbiota, a mediator of polyphenolic bioactivities, was altered significantly in MGE-treated mice; MGE increased the alpha diversity (7.14\%), Firmicutes/Bacteroidetes ratio (2-fold), relative abundance of butyrate-producing genera, and butyrate ( 3 -fold) $(P<0.05)$. Butyrate inhibited 4T1 cell proliferation and migration, suggesting butyrate contributes to MGE's anti-metastatic activities $(P<0.05)$. Conclusion Our results indicate that MGE may be an effective adjuvant therapy to reduce TNBC metastatic progression.

\section{Full Text}

This preprint is available for download as a PDF.

\section{Figures}




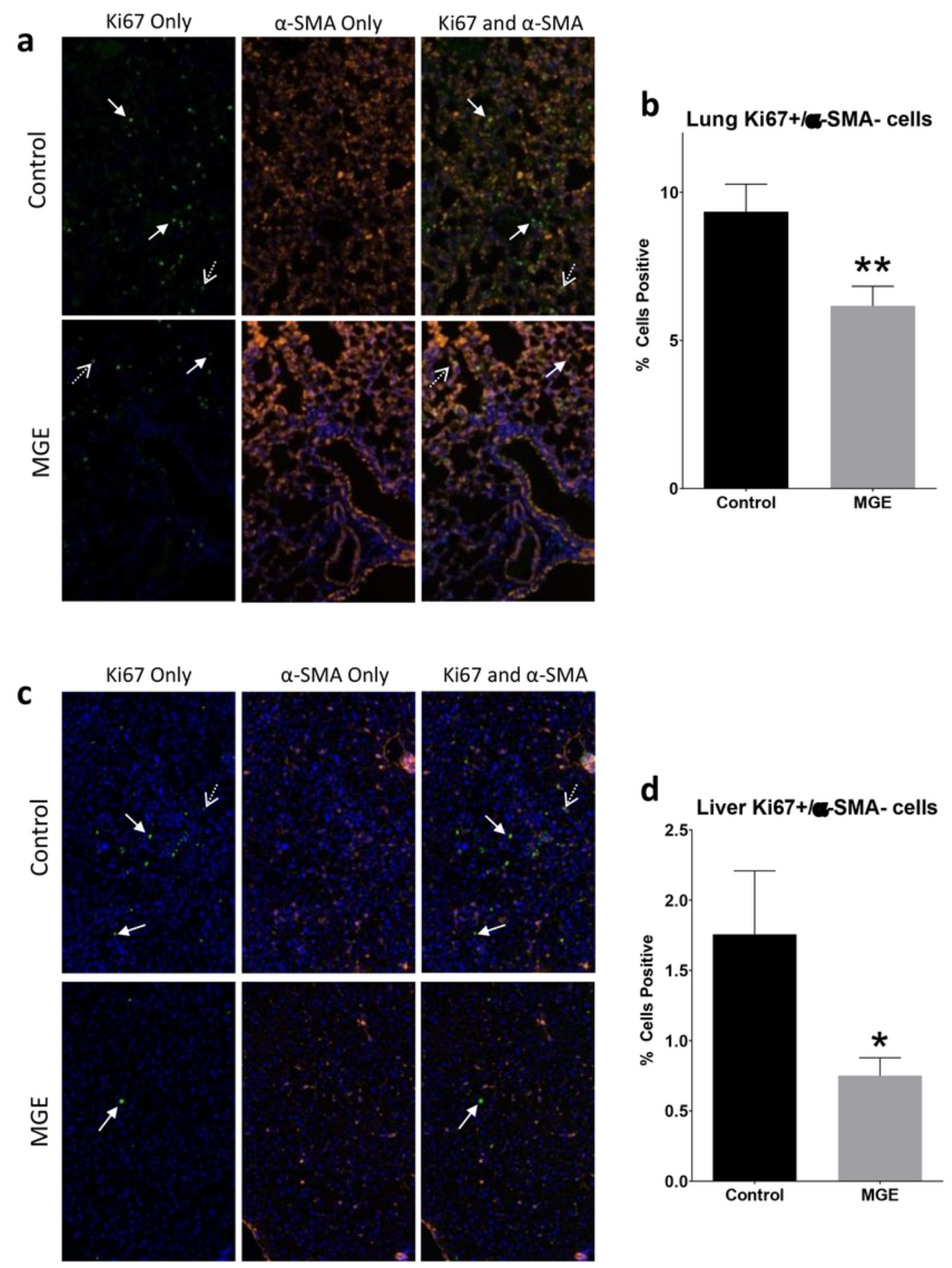

\section{Figure 1}

MGE reduces cell proliferation in lungs and livers of mice with TNBC metastasis. Representative images show lung (a) or liver (c) tissues immunostained with Ki67 (green) and/or a-SMA (orange) with DAPI (blue) as a nuclear counterstain. Solid white arrows indicate cells that are positive for Ki67 and negative for a-SMA, which were quantified as proliferative cells. Dotted white arrows indicate cells that were positive for both Ki67 and a-SMA, which may indicate proliferating fibroblasts and were excluded from 
the cell proliferation analysis. The percentage of cells Ki67+/ a-SMA-was quantified to determine nonfibroblast proliferating cells in lungs (b) and livers (d) of mice with TNBC metastasis. $n=8$; * $P<0.05$ and ** $\mathrm{P}<0.01$.
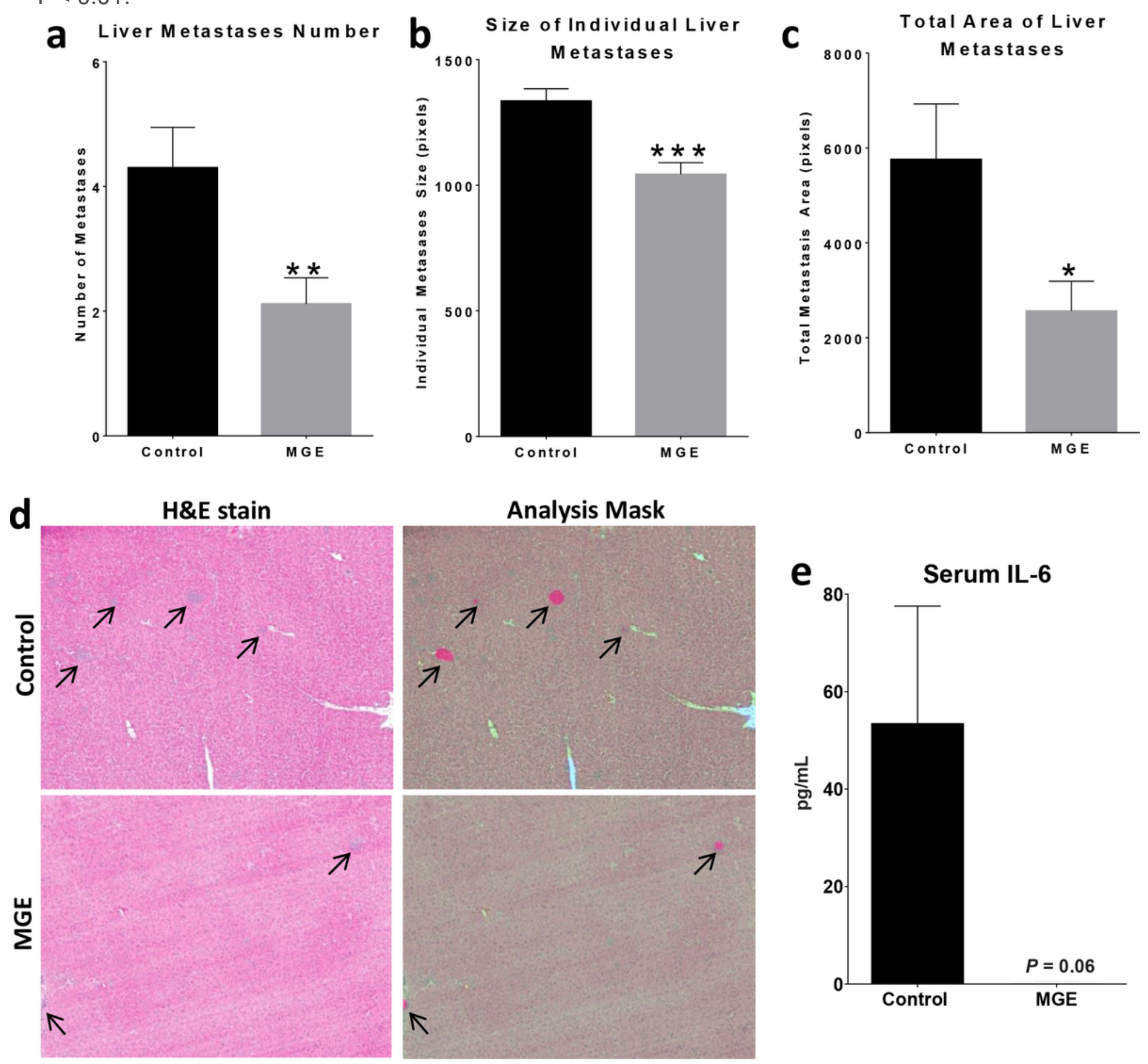

Figure 2

MGE reduces liver metastases in a metastatic mouse model of TNBC. Mouse livers were stained with hematoxylin and eosin (H\&E) to quantify the number of liver metastases (a), individual size of liver metastases (b) and total area of liver metastases (c). Representative images show H\&E stained livers with and without the analysis mask (d). The analysis mask shows healthy liver tissue as green, metastases as red, and background as cyan. Black arrows delineate metastases. Serum IL-6 was quantified by ELISA (e). $n=7-8 ; * P<0.05$, ** $P<0.01$ and *** $\mathrm{P}<0.001$. 
a
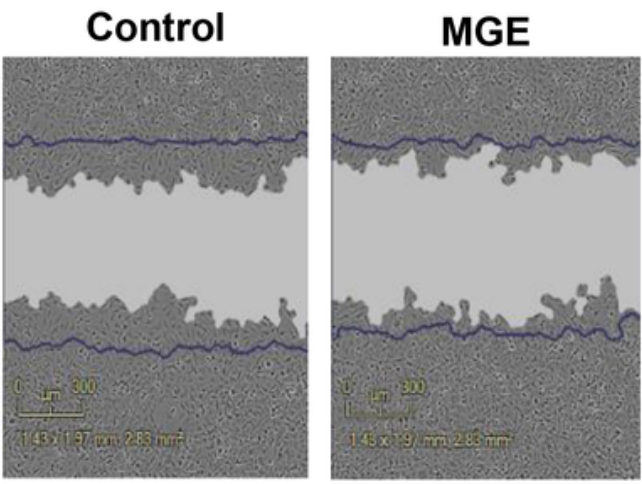

C

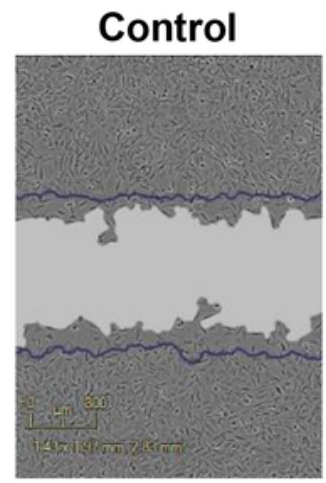

e
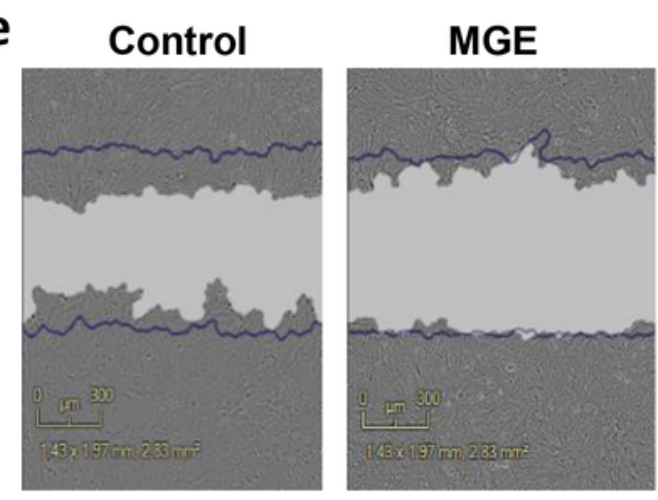

b

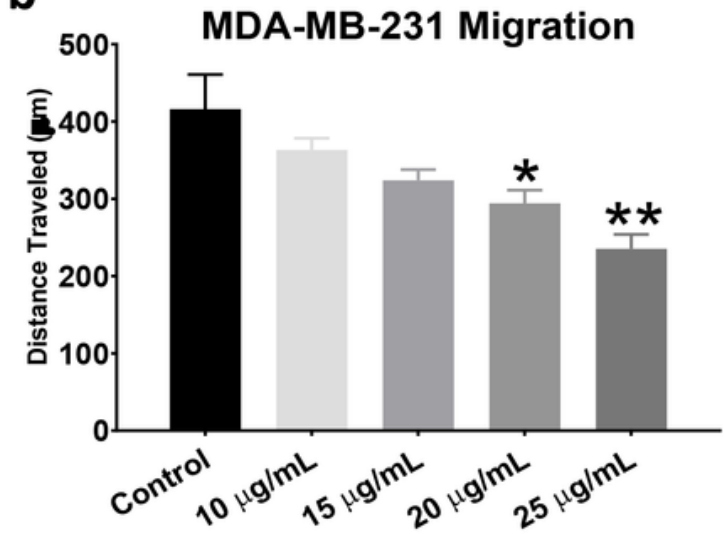

d

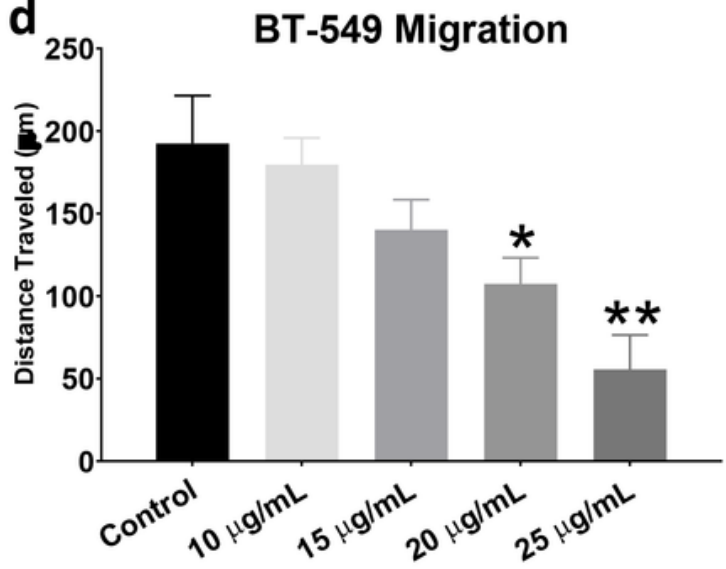

f

4T1 Migration

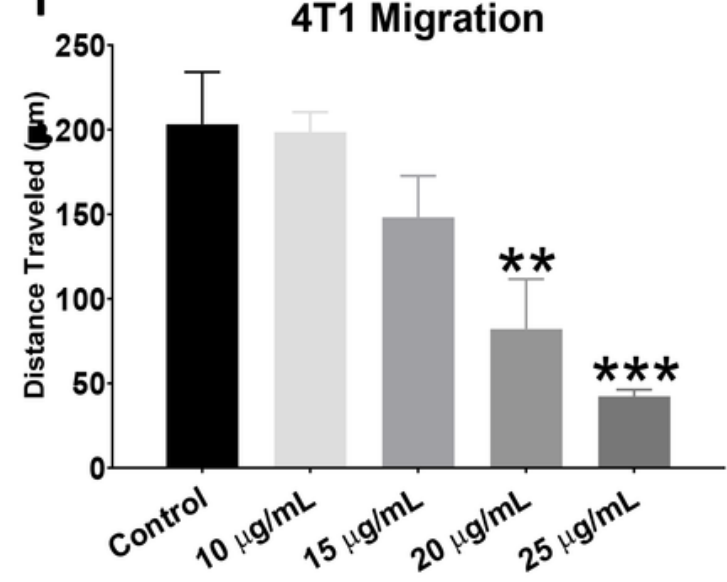

\section{Figure 3}

MGE inhibits TNBC cell migration. Confluent monolayers of MDA-MB-231 (a), BT-549 (c) or 4T1 (e) cells were uniformly scratched and treated with increasing concentrations of MGE. After $12 \mathrm{~h}$, the distance traveled by cells from the initial wound edge, shown as solid horizontal lines, to the center of the wound was quantified for MDA-MB-231 (b), BT-549 (d) and 4T1 (f) cells. Representative images show the 
distance traveled by control cells or cells treated with $20 \mu$ g phenolics $/ \mathrm{mL}$ of MGE after $12 \mathrm{~h} . \mathrm{n}=3-4$; * $\mathrm{P}<$ $0.05, * * \mathrm{P}<0.01$ and $* * * \mathrm{P}<0.001$.
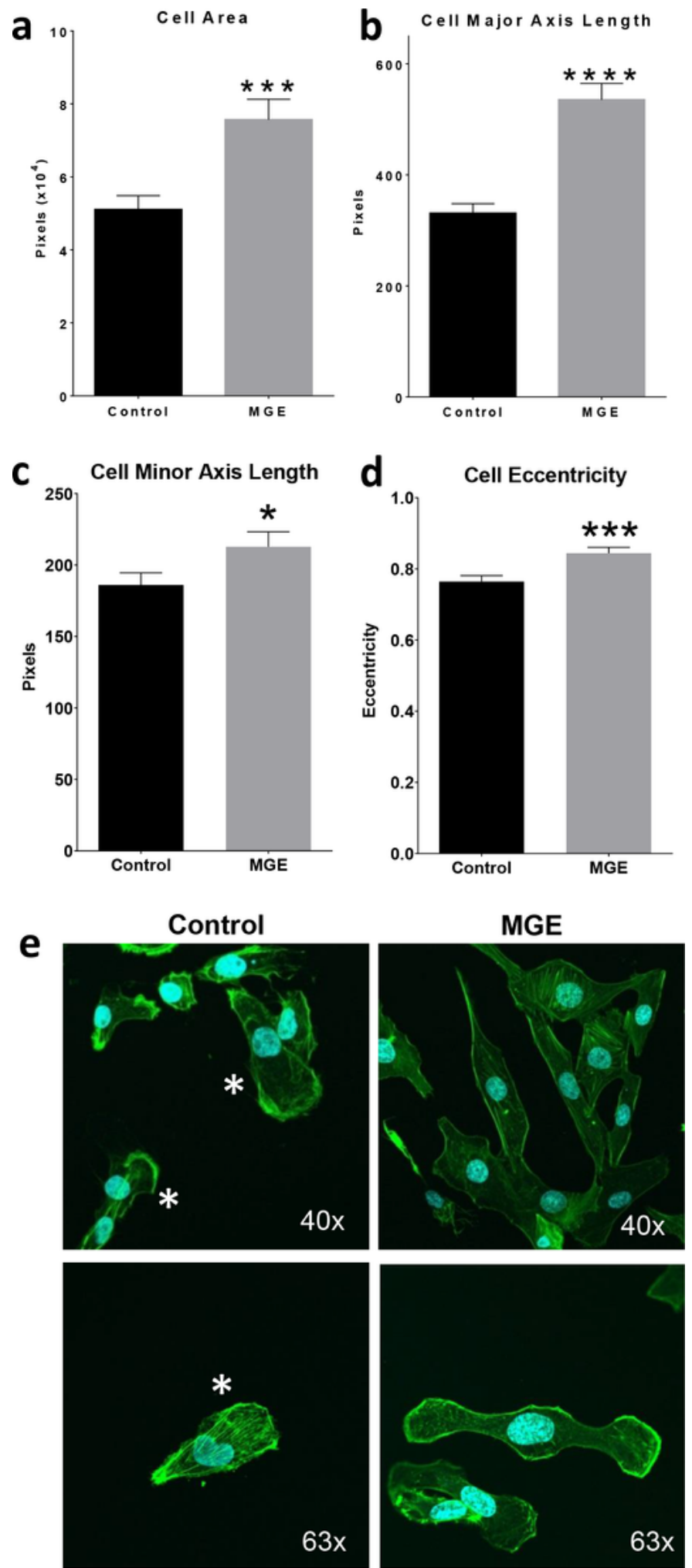

Figure 4

MGE increases MDA-MB-231 cell size and alters cytoskeletal arrangement. MDA-MB-231 cells were treated with $20 \mu \mathrm{g}$ phenolics/mL of MGE for $48 \mathrm{~h}$. Cell area (a), length (b), width (c) and eccentricity (d) were calculated. Representative confocal images (40x or $63 x$, as indicated) show untreated control or 
MGE-treated cells (e). F-actin is labeled with phalloidin as green and the nucleus with DAPI as cyan.

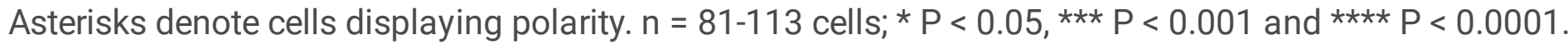
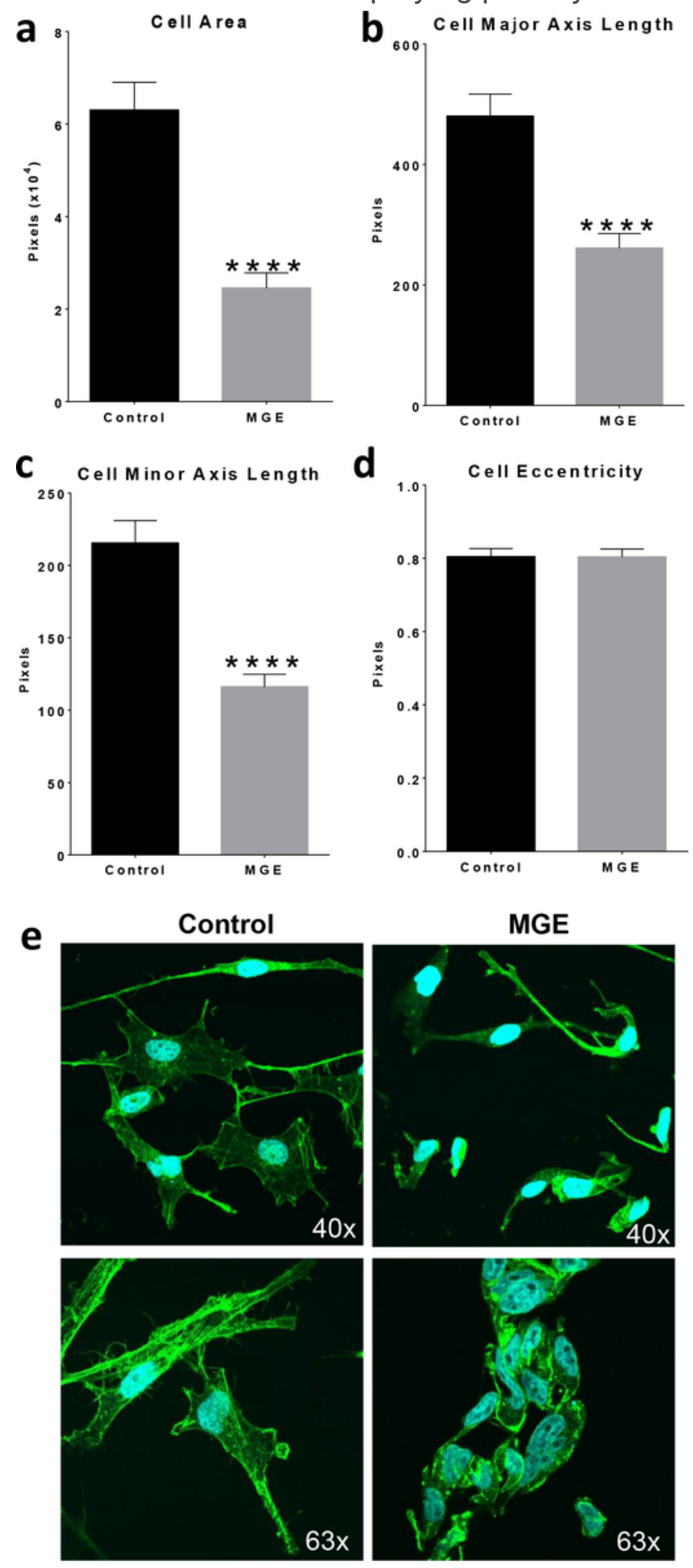

Figure 5

MGE reduces BT-549 cell size and decreases filopodia. BT-549 cells were treated with $20 \mu \mathrm{g}$ phenolics $/ \mathrm{mL}$ of MGE for $48 \mathrm{~h}$. Cell area (a), length (b), width (c) and eccentricity (d) were calculated. Representative 
confocal images (40x or $63 x$, as indicated) show untreated control cells or MGE-treated cells (e). F-actin is labeled with phalloidin as green and the nucleus with DAPI as cyan. $n=41-83$ cells; $* \star \star * ~ P<0.0001$.
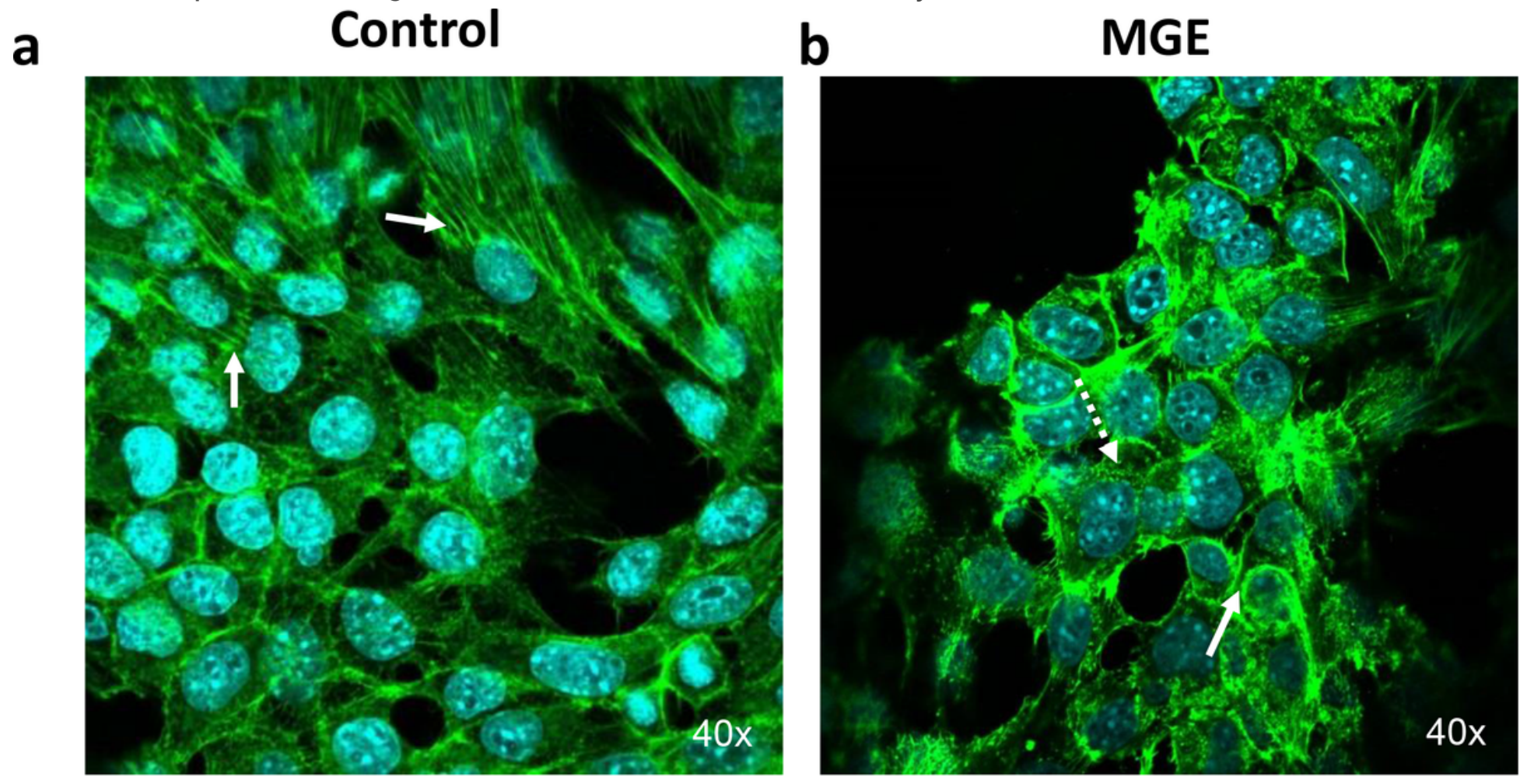

\section{Figure 6}

MGE alters 4T1 cytoskeletal arrangement. (a) Confocal images (40x) show F-actin as green and the nuclei as blue in untreated 4T1 cells or cells treated with $20 \mu \mathrm{g}$ phenolics/mL of MGE for $6 \mathrm{~h}$. In control 4T1 cells, white arrows indicate stress fibers. In MGE-treated 4T1 cells, the dotted arrow indicates punctuated F-actin and the solid arrow indicates strong peripheral F-actin. 

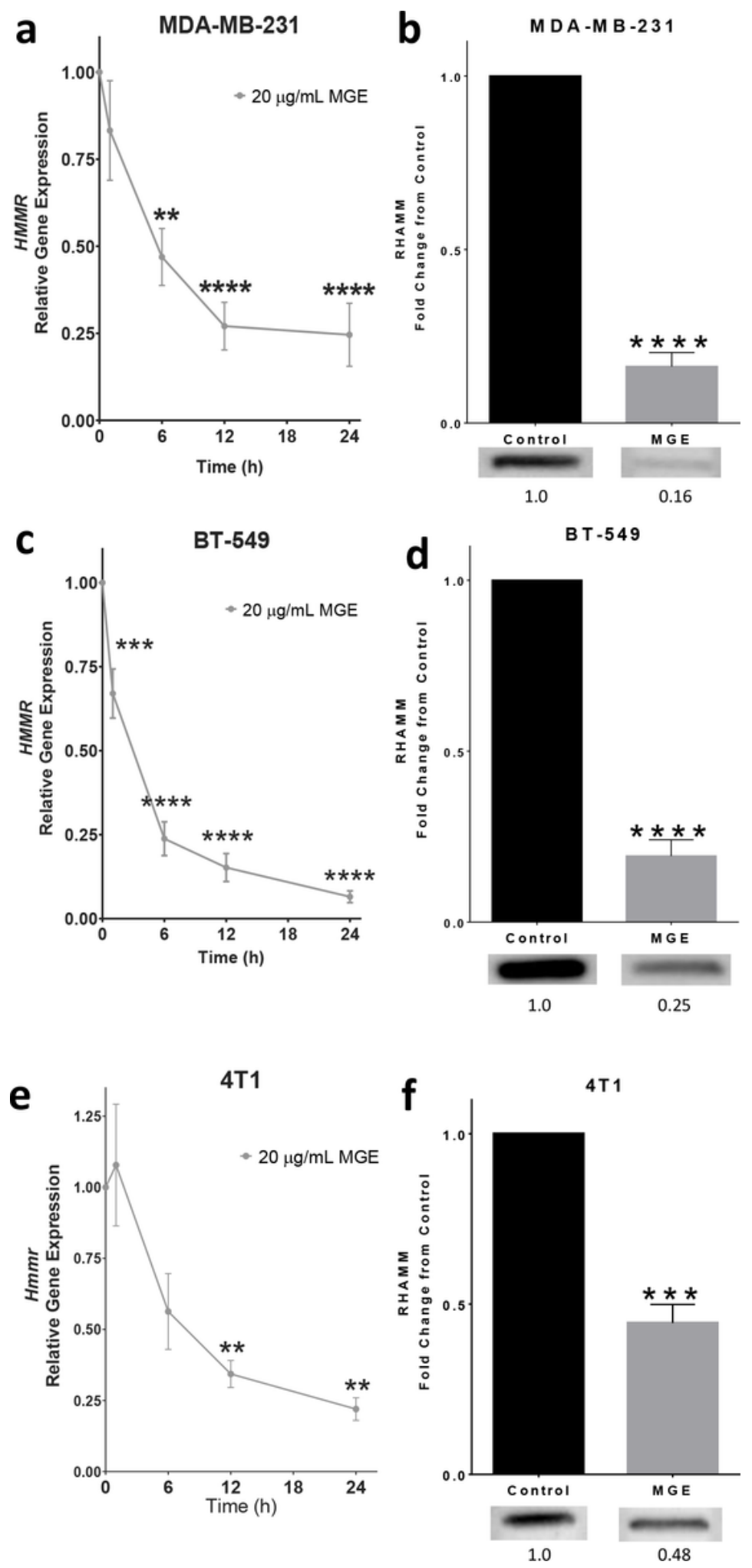

Figure 7

MGE inhibits HMMR and RHAMM in TNBC cells. MDA-MB-231 (a), BT-549 (c) and 4T1 (e) cells were treated with $20 \mu \mathrm{g}$ phenolics/mL of MGE for $1,6,12$ or $24 \mathrm{~h}$ and HMMR, the gene that encodes RHAMM, expression was measured by qRT-PCR. RHAMM protein expression was measured by western blot in MDA-MB-231 (b) and BT-549 (d) cells treated with $20 \mu \mathrm{g}$ phenolics/mL of MGE for $36 \mathrm{~h}$ or in 4T1 (f) cells treated with $20 \mu \mathrm{g}$ phenolics/mL of MGE for $12 \mathrm{~h}$. Representative bands are shown below the graphs for 
each cell type. Relative fold-change in band intensity compared to the control for RHAMM is shown below

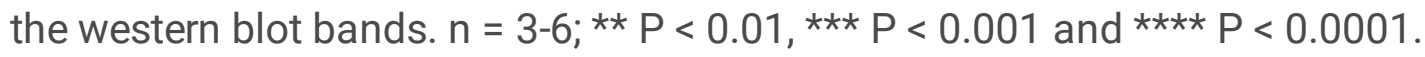
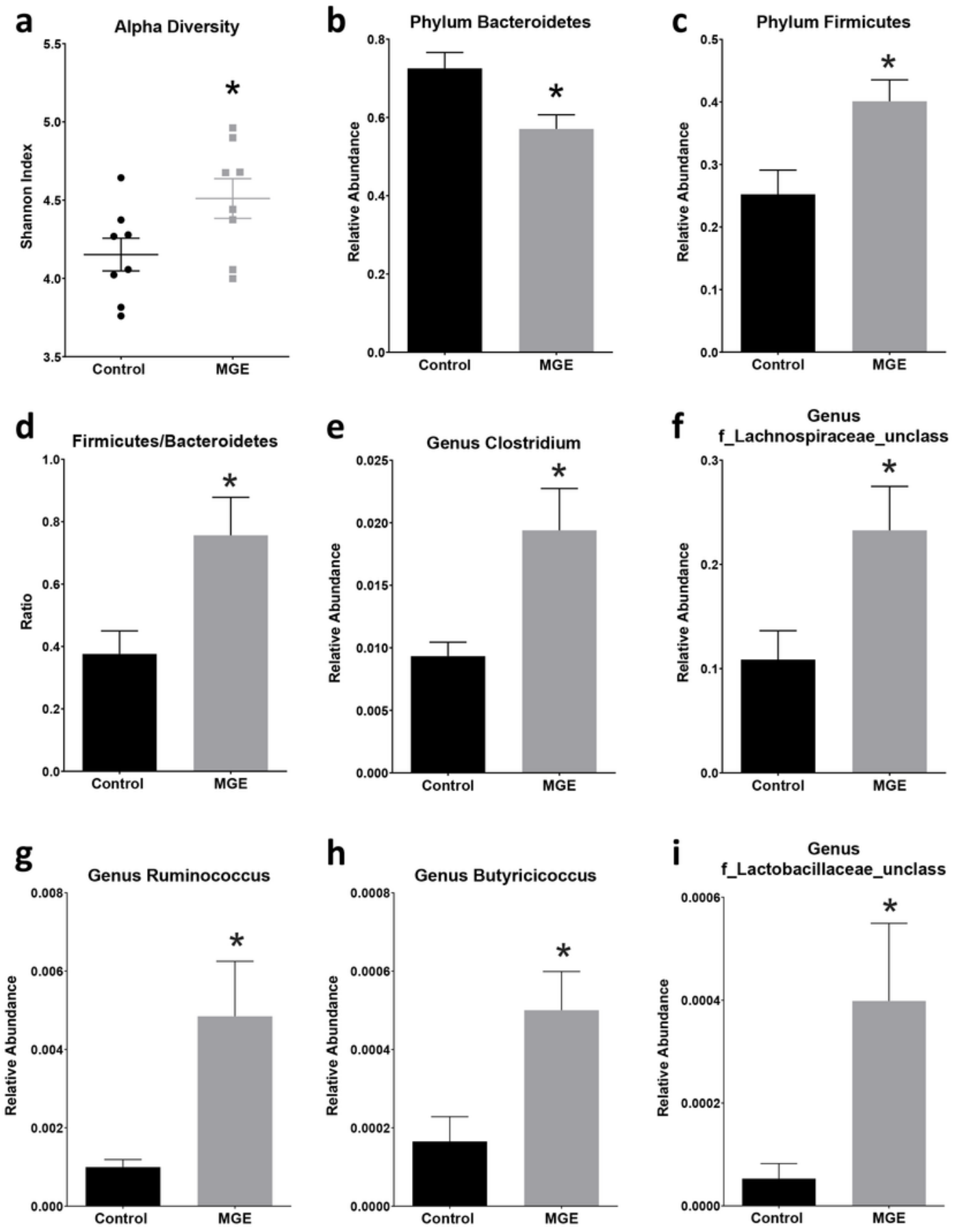

\section{Figure 8}

MGE alters gut microbiota composition in mice with metastatic TNBC. Mouse fecal samples were analyzed for gut microbiota alpha diversity (a) and the relative abundance of Firmicutes (b) and Bacteroidetes (c) phyla. The ratio of the relative abundance of Firmicutes/Bacteroidetes was quantified 
(d). The relative abundance of bacteria from the Clostridium genus (e), an unclassified genus from the Lachnospiraceae family (f), Ruminococcus genus (g), Butyricicoccus genus (h), and an unclassified genus from the Lactobacillaceae family (i) are shown. $n=8$; * $P<0.05$.
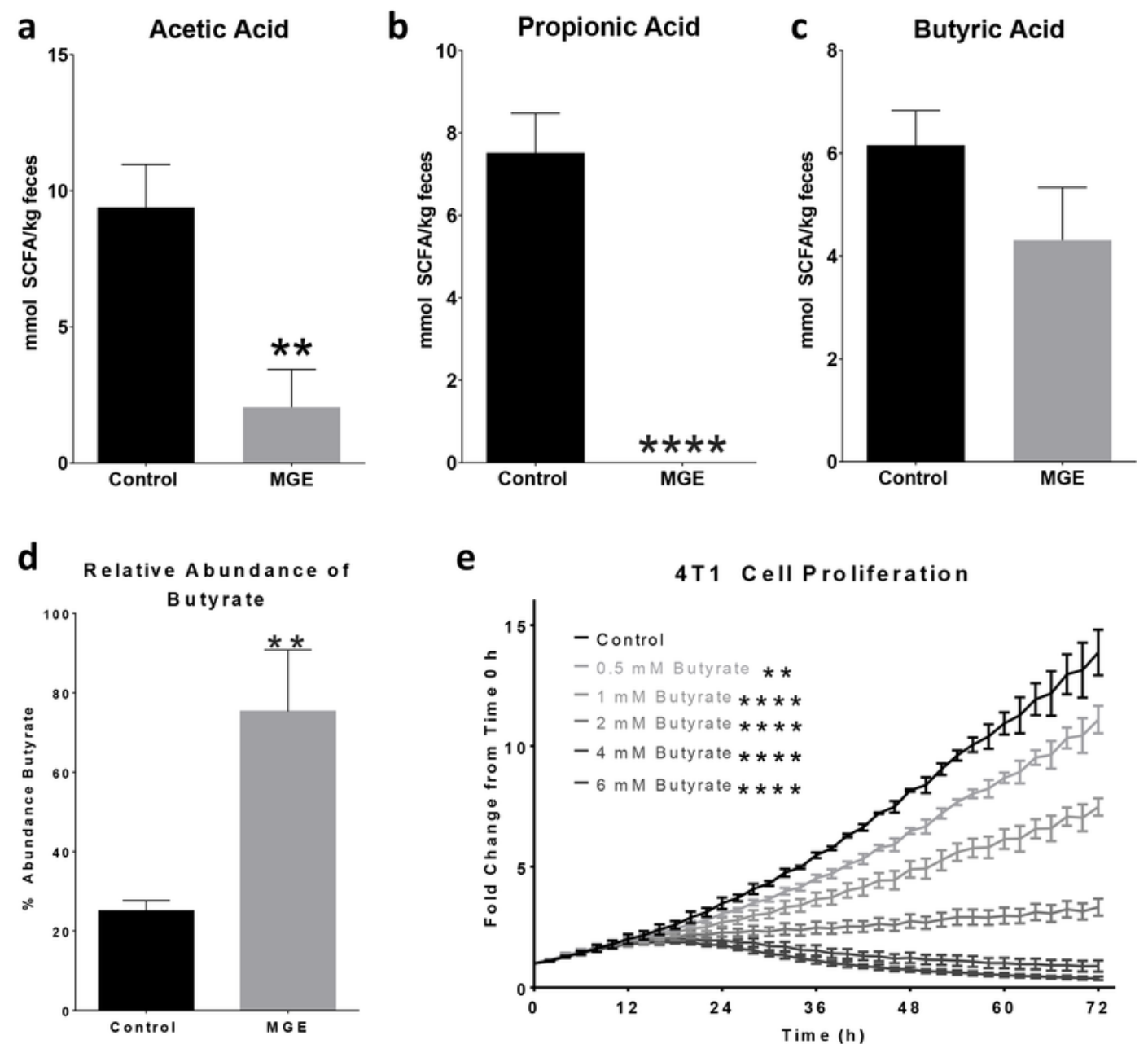

e

4T1 Cell Proliferation
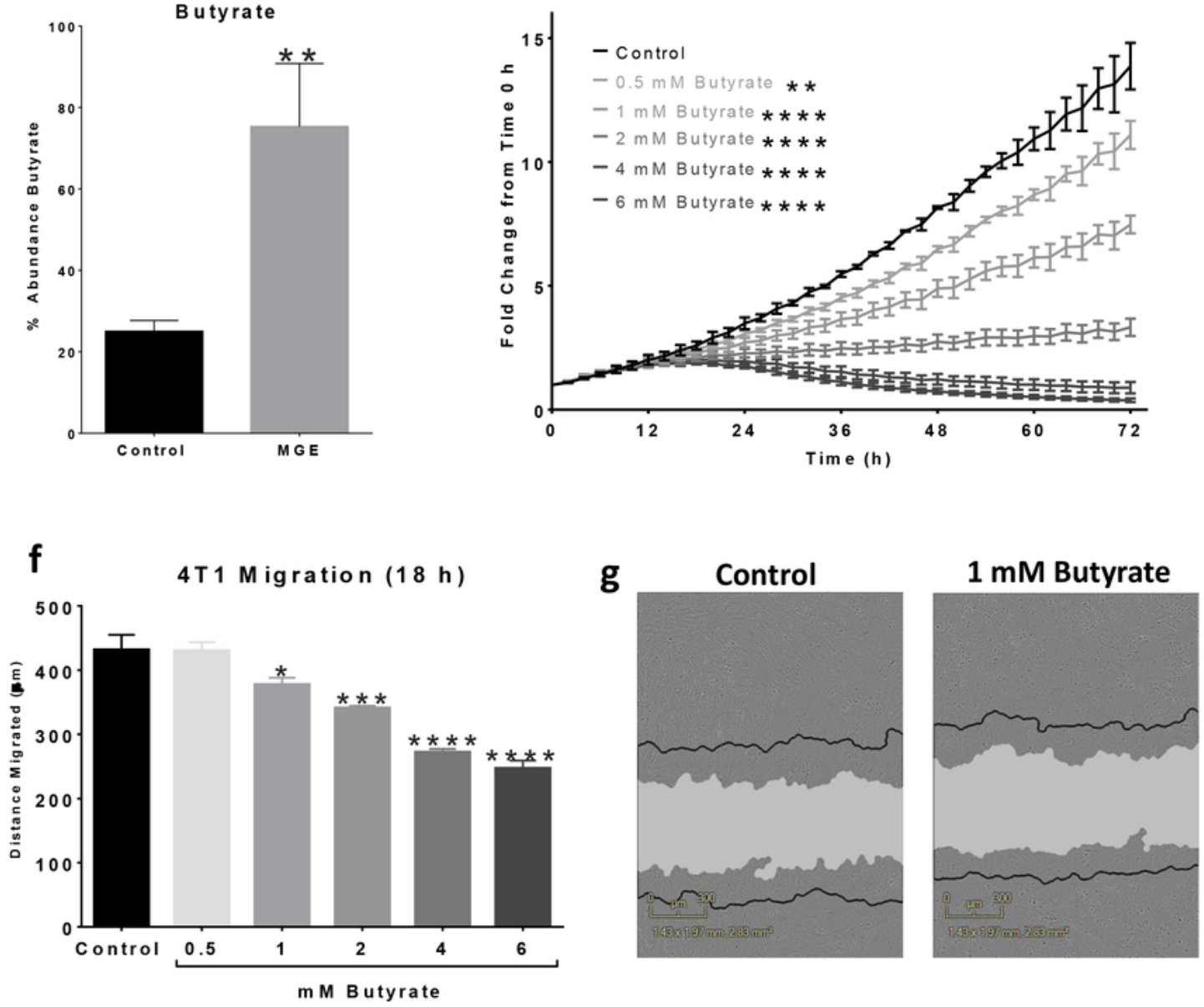

Figure 9

MGE increases the relative abundance of fecal butyrate, which inhibits 4T1 cell proliferation and migration. Short chain fatty acid composition in mouse fecal samples was determined for acetic (a), 
propionic (b) and butyric acid (c). The abundance of butyric acid relative to total acetic, propionic and butyric acids was quantified (d). 4T1 TNBC cells labeled with NucLight Red were incubated with increasing concentrations of butyrate, the butyric acid salt, and cell proliferation was measured every $2 \mathrm{~h}$ for $72 \mathrm{~h} \mathrm{(e)}$. Cell proliferation was quantified by the number of red nuclei normalized to the number of red nuclei at time $0 \mathrm{~h}$. For the scratch wound migration assay, 4T1 cells were incubated with increasing concentrations of butyrate (f). The distance traveled by cells from the initial wound edge, shown as solid horizontal lines $(\mathrm{g})$, was measured after $18 \mathrm{~h}$. Representative images of control cells or cells treated with $1 \mathrm{mM}$ of butyrate show migrated cells $18 \mathrm{~h}$ after initial wounding. $\mathrm{n}=3-8$; $* \mathrm{P}<0.05,{ }^{*} \mathrm{P}<0.01, \star \star \star \mathrm{P}<$ $0.001, \star \star \star \star ~ P<0.0001$.

\section{Supplementary Files}

This is a list of supplementary files associated with this preprint. Click to download.

- TNBCMicrobiomeSupplementalFiguresFinal.pdf

- TNBCMicrobiomeSupplementalTable1.pdf 